\title{
Study protocol for The Emory 3q29 Project: evaluation of neurodevelopmental, psychiatric, and medical symptoms in 3q29 deletion syndrome
}

Melissa M. Murphy ${ }^{1}$, T. Lindsey Burrell ${ }^{2,3}$, Joseph F. Cubells ${ }^{1,4,5}$, Roberto Antonio España ${ }^{6}$, Michael J. Gambello ${ }^{1}$, Katrina C. B. Goines ${ }^{5,6}$, Cheryl Klaiman ${ }^{2,3}$, Longchuan Li ${ }^{2,3}$, Derek M. Novacek ${ }^{6}$, Ava Papetti ${ }^{1}$,

Rossana Lucia Sanchez Russo ${ }^{1}$, Celine A. Saulnier ${ }^{2}$, Sarah Shultz ${ }^{2,3}$, Elaine Walker ${ }^{6}$ and Jennifer Gladys Mulle ${ }^{1,7^{*}}$ (D)

\begin{abstract}
Background: 3q29 deletion syndrome is caused by a recurrent hemizygous $1.6 \mathrm{Mb}$ deletion on the long arm of chromosome 3. The syndrome is rare (1 in 30,000 individuals) and is associated with mild to moderate intellectual disability, increased risk for autism and anxiety, and a 40-fold increased risk for schizophrenia, along with a host of physical manifestations. However, the disorder is poorly characterized, the range of manifestations is not well described, and the underlying molecular mechanism is not understood. We designed the Emory 3q29 Project to document the range of neurodevelopmental and psychiatric manifestations associated with 3q29 deletion syndrome. We will also create a biobank of samples from our 3q29 deletion carriers for mechanistic studies, which will be a publicly-available resource for qualified investigators. The ultimate goals of our study are three-fold: first, to improve management and treatment of 3q29 deletion syndrome. Second, to uncover the molecular mechanism of the disorder. Third, to enable cross-disorder comparison with other rare genetic syndromes associated with neuropsychiatric phenotypes.

Methods: We will ascertain study subjects, age 6 and older, from our existing registry (3q29deletion.org). Participants and their families will travel to Atlanta, GA for phenotypic assessments, with particular emphasis on evaluation of anxiety, cognitive ability, autism symptomatology, and risk for psychosis via prodromal symptoms and syndromes. Evaluations will be performed using standardized instruments. Structural, diffusion, and resting-state functional MRI data will be collected from eligible study participants. We will also collect blood from the $3 \mathrm{q} 29$ deletion carrier and participating family members, to be banked at the NIMH Repository and Genomics Resource (NRGR).

Discussion: The study of $3 q 29$ deletion has the potential to transform our understanding of complex disease. Study of individuals with the deletion may provide insights into long term care and management of the disorder. Our project describes the protocol for a prospective study of the behavioral and clinical phenotype associated with 3q29 deletion syndrome. The paradigm described here could easily be adapted to study additional CNV or single gene disorders with high risk for neuropsychiatric phenotypes, and/or transferred to other study sites, providing a means for data harmonization and cross-disorder analysis.
\end{abstract}

Keywords: 3q29 deletion, Protocol, Deep phenotyping, Rare variant

\footnotetext{
* Correspondence: jmulle@emory.edu

'Department of Human Genetics, Emory University School of Medicine,

Whitehead Research Building, 615 Michael Street, Suite 300, Atlanta, GA

30322, USA

${ }^{7}$ Department of Epidemiology, Rollins School of Public Health, Emory

University, Atlanta, GA 30322, USA

Full list of author information is available at the end of the article
}

(c) The Author(s). 2018 Open Access This article is distributed under the terms of the Creative Commons Attribution 4.0 International License (http://creativecommons.org/licenses/by/4.0/), which permits unrestricted use, distribution, and reproduction in any medium, provided you give appropriate credit to the original author(s) and the source, provide a link to the Creative Commons license, and indicate if changes were made. The Creative Commons Public Domain Dedication waiver (http://creativecommons.org/publicdomain/zero/1.0/) applies to the data made available in this article, unless otherwise stated. 


\section{Background}

As new genomic technologies are increasingly deployed in clinical settings, novel syndromes are being discovered at an astonishing pace [1]. However, articulating the range of clinical phenotypes associated with these syndromes lags behind the rate of discovery, leaving patients and clinical caretakers frustrated. This frustration is especially manifest when syndromes are associated with later-onset neuropsychiatric phenotypes such as schizophrenia, anxiety disorder, or bipolar disorder, as many are [2].

One barrier to phenotypic description is the low frequency of these syndromes, which may limit any one clinic to observing a single patient with a given syndrome. Clinical research collaboratives, such as ClinVar [3] https://www.ncbi.nlm.nih.gov/clinvar/) and the Undiagnosed Diseases Network [1] (https://undiagnosed.hms.harvard.edu/), parent-led initiatives aided by social media (e.g., Cornelia de Lange Syndrome Foundation, http:// www.cdlsusa.org/) and internet-based registries [4] (https:// simonsvipconnect.org/) have all allowed patients with similar genetic mutations to come together, even when the mutations are rare in the population. Once a critical mass of patients is assembled, a standardized phenotyping protocol can be applied in a research setting $[5,6]$. The resulting data are of critical importance in a clinical context to inform standards of care, shape expectations for patients and their families, and strengthen the relationship between families affected by these disorders and the clinicians treating them. These data are also useful for research, to inform mechanistic studies and cross-disorder comparison. Here we describe a comprehensive and systematic phenotyping protocol we have developed for use in our recently launched study to describe the phenotypes spectrum associated with 3q29 deletion syndrome.

First described in 2005, 3q29 deletion syndrome is caused by a recurrent, typically de novo, $1.6 \mathrm{Mb}$ heterozygous deletion on chromosome 3 , with an estimated incidence of approximately one in 30-40,000 births [7]. The 3q29 deletion is associated with a range of neuropsychiatric phenotypes, including elevated risk of autism spectrum disorders (ASD) [8], intellectual disability [9-11], and anxiety disorder [12]. It is also associated with a 40-fold increased risk for schizophrenia [13, 14], which may make it the single-largest molecular risk factor for schizophrenia [7].

In addition, the neuropsychiatric phenotypes associated with the 3q29 deletion appear to emerge well below the average age of risk [12]. For example, in the largest published systematic study of 3q29 deletion carriers to date, Glassford and colleagues found that $28 \%$ of 44 respondents to an online registry report at least one psychiatric diagnosis. This sample was largely pediatric (the average age of deletion carriers in this study was
11.5 years old), suggesting that more neuropsychiatric illness may manifest as this cohort moves through the age at risk [12]. Thus, the true prevalence of neuropsychiatric manifestations in 3q29 deletion syndrome may be underreported. Consistent with this notion, anecdotal reports from parents suggest that some conditions (such as ASD and anxiety disorder) are not routinely assessed in individuals with 3q29 deletion syndrome. Furthermore, additional neurodevelopmental consequences associated with schizophrenia and other neuropsychiatric disorders are not well described, the degree of heterogeneity is undocumented, and the scope of physical and medical manifestations is unclear. Direct clinical evaluation can help to address these knowledge gaps.

The present project aims to determine and quantify the behavioral and clinical phenotype of 3q29 deletion syndrome in children and adults along four dimensions, selected because of their prior association with the syndrome: anxiety, cognitive ability, autism symptomatology, and elevated prodromal features and/or psychosis. Further, we seek to document physical attributes and comorbid medical symptoms and evaluate brain structure and function. In doing so, we document a systematic approach to deep phenotyping work in 3q29 deletion syndrome and lay a foundation for subsequent studies examining the relationship between behavioral and molecular phenotypes aimed at understanding the underlying mechanisms of schizophrenia and other associated neuropsychiatric disorders.

\section{Method \\ Design}

The present study is an ongoing prospective investigation into the behavioral and clinical phenotype associated with 3q29 deletion syndrome. A biospecimen (blood) is also collected from the 3q29 deletion carrier and any participating family members and banked for use in subsequent studies investigating the molecular mechanism of the disorder. Additionally, each deletion carrier completes a medical exam to evaluate medical history and current physical attributes, and an MRI scan to examine brain structure and function.

\section{Participants \& setting}

Study participants are individuals with 3q29 deletion and their family members. Participants are eligible if the carrier has a clinically confirmed diagnosis of the 3q29 deletion, is age 6 or older, is fluent in English, and the carrier and family are willing to travel to Atlanta, GA for assessment.

All evaluations take place at the Marcus Autism Center in Atlanta, GA, an affiliate of Children's Hospital of Atlanta (CHOA), which has facilities designed for working with young people with a range of abilities and needs. The 
MRI scan takes place at the Center for Systems Imaging Core (CSIC) at Wesley Woods, which is part of Emory Healthcare.

Select self-report questionnaires are administered online prior to the visit using the REDCap web application. REDCap is approved for use in research environments to build and maintain online surveys and databases (https://www.project-redcap.org).

\section{Recruitment}

Participants are recruited from the 3q29 registry (3q29deletion.org), which has been previously described [12], and from the 3q29 private Facebook community page, initiated and maintained by the families. The study is also described on the project website (http://genome.emory.edu/3q29/). We anticipate a final sample size of 30 individuals will be characterized. This sample size was based on feasibility of recruitment as well as statistical power: as our primary analysis, we will describe the presence and severity of phenotypes, and report whether these phenotypes are elevated in 3q29 deletion carriers compared to expected age-specific population estimates. Our sample size of 30 is well powered for this analysis as it has $80 \%$ power to detect an effect size half the magnitude of the phenotype's standard deviation in the sample after applying a (conservative) Bonferroni correction for multiple testing.

When a family expresses interest in the study, they are sent copies of the study consent via email. A study team member contacts the family by phone to review the consent, answer questions, and confirm eligibility. During this initial phone screening, the team member verifies the accuracy of information from the registry (e.g., child diagnosis, age) and completes a brief eligibility screening for MRI. A hard copy of the consent and a medical record release form is mailed to the family with a postage paid return envelope. A signed consent and confirmation of diagnosis are required prior to the visit.

Within 4 weeks of the visit date, eligible participating families are contacted by a member of the MRI team to conduct a detailed eligibility screening for MRI. Participants with a contraindication for MRI are excluded from the MRI portion of the study.

\section{Visit protocol \\ Measures}

Table 1 summarizes key clinical and behavioral measures.

Anxiety Participants 18 years and younger are administered the Anxiety Disorders Interview Schedule for DSM-IV (ADIS-IV) Child and Parent versions [15], which consist of clinician-administered, semi-structured interviews that assess the child and parent's perception of the presence, duration, and degree of daily interference of specific anxiety symptoms. Anxiety symptoms are evaluated
Table 1 Study Measures by Schedule and Domain Assessed

\begin{tabular}{|c|c|c|}
\hline Domain & Age & Measure \\
\hline \multirow[t]{2}{*}{ Anxiety } & $\leq 17$ Years & $\begin{array}{l}\text { Anxiety Disorders Interview } \\
\text { Schedule for DSM -IV (ADIS-IV) } \\
\text { Child Interview Schedule } \\
\text { Parent Interview Schedule }\end{array}$ \\
\hline & $\geq 18$ Years & $\begin{array}{l}\text { Kiddie Schedule for Affective } \\
\text { Disorders and Schizophrenia } \\
\text { (K-SADS) or Structured Clinical } \\
\text { Interview for DSM-V - Research } \\
\text { Version (SCID-5-RV) - Module F } F^{f}\end{array}$ \\
\hline \multirow[t]{2}{*}{ Autism } & All & $\begin{array}{l}\text { Autism Diagnostic Interview } \\
\text { - Revised (ADI-R) }\end{array}$ \\
\hline & All & $\begin{array}{l}\text { Autism Diagnostic Observation } \\
\text { Schedule, 2nd ed. (ADOS-2) } \\
\text { Modules 1, 2, 3, or } 4\end{array}$ \\
\hline \multirow{5}{*}{$\begin{array}{l}\text { Cognitive Ability \& } \\
\text { Adaptive Function }\end{array}$} & $\leq 17$ Years & Differential Ability Scales, 2nd ed. (DAS-II) \\
\hline & $\geq 18$ Years & $\begin{array}{l}\text { Wechsler Abbreviated Scale of } \\
\text { Intelligence, 2nd ed. (WASI-II) }\end{array}$ \\
\hline & All & $\begin{array}{l}\text { Vineland Adaptive Behavior } \\
\text { Scales, 3rd ed., Parent/ } \\
\text { Caregiver Form }\end{array}$ \\
\hline & $\begin{array}{l}\leq 18 \text { Years } \\
>18 \text { Years }\end{array}$ & $\begin{array}{l}\text { Behavioral Rating Inventory } \\
\text { of Executive Function, } \\
\text { 2nd ed. (BRIEF-2) }{ }^{b} \\
\text { Behavioral Rating Inventory } \\
\text { of Executive Function-Adult } \\
\text { Version (BRIEF-A) }{ }^{b}\end{array}$ \\
\hline & All & $\begin{array}{l}\text { Beery-Buktenica Test of Visual Motor } \\
\text { Integration, 6th Edition (VMI-6) }\end{array}$ \\
\hline \multirow[t]{2}{*}{$\begin{array}{l}\text { Prodromal Symptoms } \\
\text { \& Psychosis }\end{array}$} & $\begin{array}{l}\geq 8 \text { Years } \\
\text { All }\end{array}$ & $\begin{array}{l}\text { Structured Interview for Psychosis-Risk } \\
\text { Syndromes (SIPS) } \\
\text { Pre-interview, c, d }\end{array}$ \\
\hline & $\geq 18$ Years & $\begin{array}{l}\text { Structured Clinical Interview } \\
\text { for DSM-V - Research Version } \\
\text { (SCID-5-RV) - Module B/C }\end{array}$ \\
\hline \multirow[t]{2}{*}{ General Psychopathology } & $\begin{array}{l}\leq 21 \text { Years }^{f} \\
\leq 21 \text { Years }^{f} \\
\text { All } \\
\text { All }\end{array}$ & $\begin{array}{l}\text { Kiddie Schedule for Affective } \\
\text { Disorders and Schizophrenia } \\
\text { (K-SADS) } \\
\text { Child Interview } \\
\text { Parent Interview } \\
\text { Cross Cutting Survey }{ }^{\text {b, c, d }} \\
\text { Pre-interview }{ }^{\text {b, d }}\end{array}$ \\
\hline & $\geq 22$ Years & $\begin{array}{l}\text { Structured Clinical Interview } \\
\text { for DSM-V - Research Version } \\
\text { (SCID-5-RV) - Modules } \\
\text { A, D, G, H, I, K }\end{array}$ \\
\hline $\begin{array}{l}\text { Anthropomorphic } \\
\text { Measures/Medical } \\
\text { Exam }\end{array}$ & All & $\begin{array}{l}\text { Study generated Medical } \\
\text { History Form and Vitals } \\
\& \text { Review of Systems-based } \\
\text { Intake Form }\end{array}$ \\
\hline $\begin{array}{l}\text { Magnetic Resonance } \\
\text { Imaging (MRI) }\end{array}$ & All & $\begin{array}{l}\text { See MRI description in } \\
\text { Methods section }\end{array}$ \\
\hline Family Demographics ${ }^{b}$ & All & $\begin{array}{l}\text { Study generated } \\
\text { Demographics Form }\end{array}$ \\
\hline
\end{tabular}

aThe child interview portion is administered based upon the child's capacity to engage in the interview. ${ }^{\mathrm{b}} \mathrm{Administered} \mathrm{prior} \mathrm{to} \mathrm{visit} \mathrm{online} \mathrm{via}$ REDCap or publisher web application (BRIEF-2, BRIEF-A, Vineland-3). 'Completed by $3 \mathrm{q} 29$ deletion carriers when appropriate. ${ }^{\mathrm{d}}$ Administered to all participants prior to visit to guide clinicians in identifying areas of focus during interview. ${ }^{~} T o$ avoid overlap with the ADIS and ADI-R/ADOS, the anxiety and autism sections of the KSADS are omitted. 'Participants 1821 years are administered the KSADS (including anxiety section) in lieu of the SCID-V-R, if level of communication abilities and emotional status suggest the need for parental perspective to ensure a thorough evaluation 
based on the Diagnostic and Statistical Manual of Mental Disorders - IV (DSM-IV). These comprehensive interviews assess all anxiety disorder diagnoses observed in young children. Moreover, interviewers are trained to reliability and are knowledgeable about the distinction between autism symptomatology and DSM-IV anxiety disorders. The child interview portion is administered based upon the child's capacity to engage in the interview. If the child's communication abilities and emotional status are not adequate to participate in the interview, it is omitted. For participants over 18 years of age, anxiety is assessed using the Kiddie Schedule for Affective Disorders and Schizophrenia (K-SADS) or Structured Clinical Interview for DSM-5 Disorders (SCID-5)-Module F (described subsequently in General Psychopathy section) based upon participant communication abilities.

Autism Spectrum disorder The Autism Diagnostic Interview, Revised (ADI-R) [16] and Autism Diagnostic Observation Schedule, Second Edition (ADOS-2) [17] are used to help evaluate for Autism Spectrum Disorder (ASD). The ADI-R is a semi-structured comprehensive parent/caregiver interview designed to evaluate early developmental history and current and lifetime presentation of autism symptomatology.

The ADOS-2 is a diagnostic, semi-structured clinical assessment that directly observes for behaviors associated with ASD in the areas of social communication, play and interaction, and restrictive and repetitive behaviors. It consists of a clinical procedure that places a child in unstructured, social and playful situations. During this assessment, the individual receives no instructions or guidelines indicating how to respond. In this way, a sample of naturalistic, social and communicative behaviors is obtained. The ADOS-2 consists of modules based upon age and language level: nonverbal or minimally verbal (Module 1), uses words and phrases (Module 2), speaks in complex sentences (e.g., connect ideas using "and" or "but"; Module 3), and verbally fluent older adolescents or adults (Module 4).

In anticipation of ADOS-2 and ADI-R administration, each participant's 3q29 Registry data from four parent-report screening measures are downloaded, scored, and shared with clinicians: Achenbach Behavior Checklist (CBCL/ ABCL) [18-20], Social Responsiveness Scale-2 (SRS-2) [21], Social Communication Questionnaire (SCQ) [22], and Autism Spectrum Screening Questionnaire (ASSQ) [23]. The Achenbach checklists are commonly used measures to assess a range of social, emotional, and behavior problems. The SRS-2, SCQ, and ASSQ are widely used screening tools to assess aspects of the social and communication impairments typically associated with ASD.

IQ and adaptive behavior Overall cognitive ability is assessed using the Differential Ability Scales - 2nd edition (DAS-II; 6-17 years) [24] or Wechsler Abbreviated Scale of Intelligence, Second Edition (WASI-II; 18 years and older) [25]. Along with cognitive ability, deficits in adaptive behavior are required for diagnosis of intellectual disability. Adaptive behavior, defined as the performance of day-to-day activities that are necessary for self-care and to get along with others, is assessed using The Vineland Adaptive Behavior Scales, Third Edition, Parent Form (Vineland-3) [26].

The DAS-II assesses cognitive skills in three domains: Verbal Reasoning, Nonverbal Reasoning, and Spatial Reasoning. In addition, a General Conceptual Ability Score (GCA) is reported. The WASI-II provides a brief but reliable measure of cognitive ability in the areas of Verbal Comprehension and Perceptual Reasoning [25], with an overall Full-Scale IQ, and verbal and performance IQs obtained.

The Vineland-3 is a standardized parent interview that assesses skills an individual does independently on a daily basis in the areas of Communication, Daily Living Skills, Socialization, and Motor Skills. For the present study, the Vineland-3 is administered and scored using the Pearson q-Global online web application available through the publisher.

Visual-motor ability Visual and motor abilities are evaluated using the three developmental tests of the Beery-Buktenica Developmental Test of Visual-Motor Integration - 6th edition (VMI-6) [27]. Together these three tests require participants to copy (Visual Motor Integration), identify (Visual Perception), and trace (Motor Coordination) each of 28 abstract designs of increasing complexity.

Executive function Executive functions are assessed using the parent/informant forms of the Behavioral Rating Inventory of Executive Function, 2nd edition (BRIEF-2) [28] for participants up to 18 years old or the Behavioral Rating Inventory of Executive Function-Adult Version (BRIEF-A) [29] for participants over 18 years old. These inventories take $10 \mathrm{~min}$ (BRIEF-2) to $15 \mathrm{~min}$ (BRIEF-A) to complete and ask the informant to rate child behaviors associated with self-control and problem-solving skills along nine dimensions of executive functioning: inhibiting distractions, self-monitoring, shifting, emotional control, initiation, working memory, planning, organization, task monitoring. Scores within and over a specific threshold are considered "At Risk" or "Clinically Significant," respectively. For the present study, the BRIEF-2 and BRIEF-A are administered and scored using the PARiConnect online web application available through the publisher.

Prodromal symptoms and psychosis The Structured Interview for Psychosis-Risk Syndromes (SIPS) [30] 
assesses the presence, duration, and severity of subthreshold symptoms of psychosis. The SIPS is a reliable and valid semi-structured interview used to assess prodromal symptoms of psychosis and to determine if individuals meet criteria for an Attenuated Psychotic Syndrome, which is assessed across five different positive symptom domains: unusual thought content/delusional ideas, suspiciousness/persecutory ideas, grandiose ideas, perceptual abnormalities/hallucinations, and disorganized communication. This measure also yields ratings for six negative, four disorganized, and four general psychiatric symptoms. The SIPS is one of the primary instruments used in the North American Prodromal Longitudinal Study (NAPLS), enabling cross-comparison between these two studies [31].

Prior to the visit, the pre-interview questions for the SIPS are sent via REDCap to the parents and 3q29 deletion carrier (if over 18 years, and able to complete questionnaires). The SIPS-pre-interview questions take about 15-20 min to complete and ask about general medical and psychiatric history, and current thoughts, feelings, and perceptual experiences.

General psychopathology The Kiddie-Schedule for Affective Disorders and Schizophrenia (K-SADS) [32] assesses general psychopathology among participants younger than 18 years. This semi-structured interview assesses both the child and parent's perception of the presence, duration, and degree of daily interference of symptoms associated with affective and psychotic disorders. Symptoms are evaluated based on the Diagnostic and Statistical Manual of Mental Disorders - V (DSM-V) used by psychologists and other mental health providers to differentially diagnose affective or psychotic symptoms that interfere with daily functioning. Just as with the ADIS, the child interview portion is administered based upon the child's capacity to engage in the interview.

The KSADS cross-cutting survey and pre-interview questions are sent to the parents and 3q29 deletion carriers (if applicable) before the visit via REDCap. Together the questionnaires take about 25-35 min and ask about reactions to experiences (cross-cutting) and general behavior and development (pre-interview).

For participants 18 years and older, general psychopathology, including anxiety, is assessed using the KSADS (if level of communication abilities and emotional status suggest the need for parental perspective to ensure a thorough evaluation) or Structured Clinical Interview for DSM-5 Disorders Research Version (SCID-5-RV) [33]. The SCID-5-RV is a semi-structured interview that assesses an individual's perception of the presence, duration, and degree of daily interference of symptoms associated with various psychological disorders. Symptoms are evaluated based on the Diagnostic and Statistical Manual of Mental Disorders - Fifth Edition (DSM-5) used by psychologists and other mental health providers to differentially diagnose disorders including affective, anxiety, and psychotic disorders that interfere with daily functioning. Modules administered include: A (mood episodes), B/C (psychiatric disorders), D (mood disorders), F (anxiety), G (obsessive-compulsive and related disorders), $\mathrm{H}$ (sleep disorders), I (feeding and eating disorders), and $\mathrm{K}$ (externalizing disorders, including ADHD).

Anthropomorphic measures/medical exam The medical exam is conducted by trained clinical geneticists (RS and MG) to assess medical history (e.g., birth weight, feeding problems), evaluate physical stature (e.g., height, weight, BMI, head circumference, Tanner Stage), and take high resolution photographs for rating by a dysmorphologist to determine whether a characteristic facial phenotype exists. A detailed medical history is completed online by the parent prior to the visit using the REDCap Survey feature. The clinical geneticists review this with the families in person at the study visit. Parents are also asked to sign a medical records release, which is used to request participant medical records, including growth trajectories and detailed medical history.

MRI MRI data are collected on a Siemens Magnetom Prisma $3 \mathrm{~T}$ scanner at CSIC using a 32-channel head coil. High-resolution structural MRI, diffusion weighted imaging, and resting-state functional MRI data are acquired.

Structural MRI T1-weighted and T2-weighted high-resolution structural images are acquired. T1-weighted images are acquired using a T1-weighted 3D MPRAGE sequence with the following parameters: $\mathrm{TE}=2.24 \mathrm{~ms}$, $\mathrm{TR}=$ $2400 \mathrm{~ms}$, flip angle $=8^{\circ}$, matrix $=320 \times 320, \quad F O V=$ $256 \times 256 \mathrm{~mm}, 208$ sagittal slices, $0.8 \mathrm{~mm}$ isotropic resolution, bandwidth $=210 \mathrm{~Hz} /$ pixel. A GRAPPA factor of 2 is used with no phase oversampling and two repetitions. The total scan duration is $13 \mathrm{~min}, 16 \mathrm{~s}$. A 3D T2-weighted Sampling Perfection with Application optimized Contrast using different angle Evolutions (SPACE) sequence is employed. Details of the protocol are: $\mathrm{TE}=563 \mathrm{~ms}$, $\mathrm{TR}=3200 \mathrm{~ms}$, bandwidth $=745 \mathrm{~Hz} /$ pixel, FOV $=256 \times$ $240 \times 256 \mathrm{~mm}^{3}$, sagittally acquired, $0.8 \mathrm{~mm}$ isotropic resolution. A GRAPPA factor of 2 is used with no phase oversampling and two repetitions. The total scan duration is $11 \mathrm{~min}$.

Diffusion weighted imaging (DWI) DWI data are acquired using a high-angular-resolution-diffusion imaging (HARDI) protocol ${ }^{96}$ and multiband technique to reduce scan time while still acquiring data with high spatial and angular resolution ${ }^{97,98}$. Imaging parameters 
are: $\mathrm{TR}=3222 \mathrm{~ms}, \mathrm{TE}=89.2 \mathrm{~ms}$, multiband factor $=4$, $\mathrm{FOV}=212 \times 184 \mathrm{~mm}^{2}, \quad$ matrix $=106 \times 97, \quad b=0, \quad 1000$, $2000 \mathrm{~s} / \mathrm{mm}^{2}, 128$ diffusion directions evenly distributed on the two shells, spatial resolution $=2 \mathrm{~mm}$ isotropic, 66 slices covering the whole brain, for a total of 10 averages of b0s. B0s in two shells have opposite phase encoding directions for removing susceptibility-related distortion present in $\mathrm{dMRI}^{68}$. Total scan duration is 8 mins.

Resting-state functional MRI Resting-state functional MRI data are acquired using a multiband T2*-weighted EPI imaging sequence with the following parameters: $\mathrm{TE}=33 \mathrm{~ms}, \quad \mathrm{TR}=720 \mathrm{~ms}$, flip angle $=53^{\circ}$ to match Earnest angle, matrix $=84 \times 84, \mathrm{FOV}=210 \times 210 \mathrm{~mm}$, spatial resolution $=2.5 \mathrm{~mm}$ isotropic, 54 slices covering the whole brain, bandwidth $=2290 \mathrm{~Hz} /$ pixel, echo spacing $=0.58 \mathrm{~ms}$. Data are collected across two runs, for a total of 1140 volumes. Total scan duration is $14 \mathrm{~min}$, 30 s. 20 volumes of fMRI data are collected in the opposite phase encoding direction at the end of each fMRI run to correct for susceptibility-related distortion ${ }^{94}$.

Family demographics A study-designed demographics questionnaire is used to gather information about the participant and family, including family size, race/ethnicity, parent education and employment, and household income. The questionnaire takes about $5 \mathrm{~min}$ to complete and is sent to the parent prior to the visit using the REDCap survey feature.

\section{Procedures}

\section{Visit preparation}

Visits are scheduled at least 2-3 months in advance to allow time to coordinate clinical team availability and make travel arrangements. To prepare, within a month of the visit date, an email link to the pre-visit self-report questionnaires (see Table 1) is sent from REDCap. When appropriate, participants over 18 years old are also asked to complete applicable questionnaires (see Table 1). The questionnaires include the family demographic form, pre-interview questions for the SIPS and KSADS, KSADS Cross Cutting survey, medical history form, and a brief survey of prodromal symptoms [34] (if not already completed in the registry). Once a family completes the questionnaires, they are sent links to complete the Vineland-3 and age-appropriate BRIEF (if applicable).

Families are also invited to provide any current Individualized Education Plans (IEPs) and medical, psychological, or psycho-educational testing reports that could help the team in assessing the participant.

Approximately one week prior to the visit, clinical team members receive the participant dossier that includes questionnaire data and reports provided by the family to help prepare for the visit. Also included are select participant data from behavioral measures completed as part of the 3q29 Registry [5]: CBCL, SRS-2, SCQ, and ASSQ, survey of prodromal symptoms [34].

\section{Visit execution}

Figure 1 summarizes the study protocol. Participant age, verbal ability, and presence of active schizophrenia or psychosis are used to determine testing schedule and measures administered. For example, a verbally fluent 8-year-old child would complete Visit Schedule C; a 21-year-old with no evidence of active psychosis would complete Visit Schedule F.

Responses in the registry and confirmed during the initial phone screening are used to determine verbal ability (based upon ADOS-2 requirements, described subsequently) and presence of schizophrenia or psychosis. Sometimes conversation with the family suggests the presence of unconfirmed or undiagnosed psychosis for participants under 18 years old. In these cases, the visit schedule is modified to include individual psychiatric evaluation by the team psychiatrist (JFC).

Testing takes place over two week days. To ensure informed consent prior to testing, a study team member reviews the consent form in detail with the family and answers questions upon their arrival at the testing center. When applicable, child assent is obtained. With parent or participant permission, behavioral assessments are video and audiotaped for future reference. The medical history is audiotaped.

Blood sample collection All participants and family members are asked to provide 4-5 vials (about 3 tablespoons) of blood from a vein in the arm. Blood is drawn by a trained pediatric phlebotomist (J J-D). To avoid biasing the results of assessments due to a negative experience with a blood draw, the blood sample is scheduled towards the end of the first day's assessments. Remote blood draws are arranged locally for family members who are unable to travel to Atlanta for the evaluation.

MRI The MRI scan lasts 30-120 min with breaks as needed. During the scan, participants are asked to view video clips of people talking and interacting. An 8-min segment is also required where the participant simply lays still with no video input.

\section{Visit wrap-up}

At the end of the second visit day, the clinical team convenes privately to discuss results, impressions, and recommendations. Following this clinical case conference, the team meets with the family to share their impressions: preliminary results of evaluations, the relative strengths and challenges of the person with $3 \mathrm{q} 29$, and 


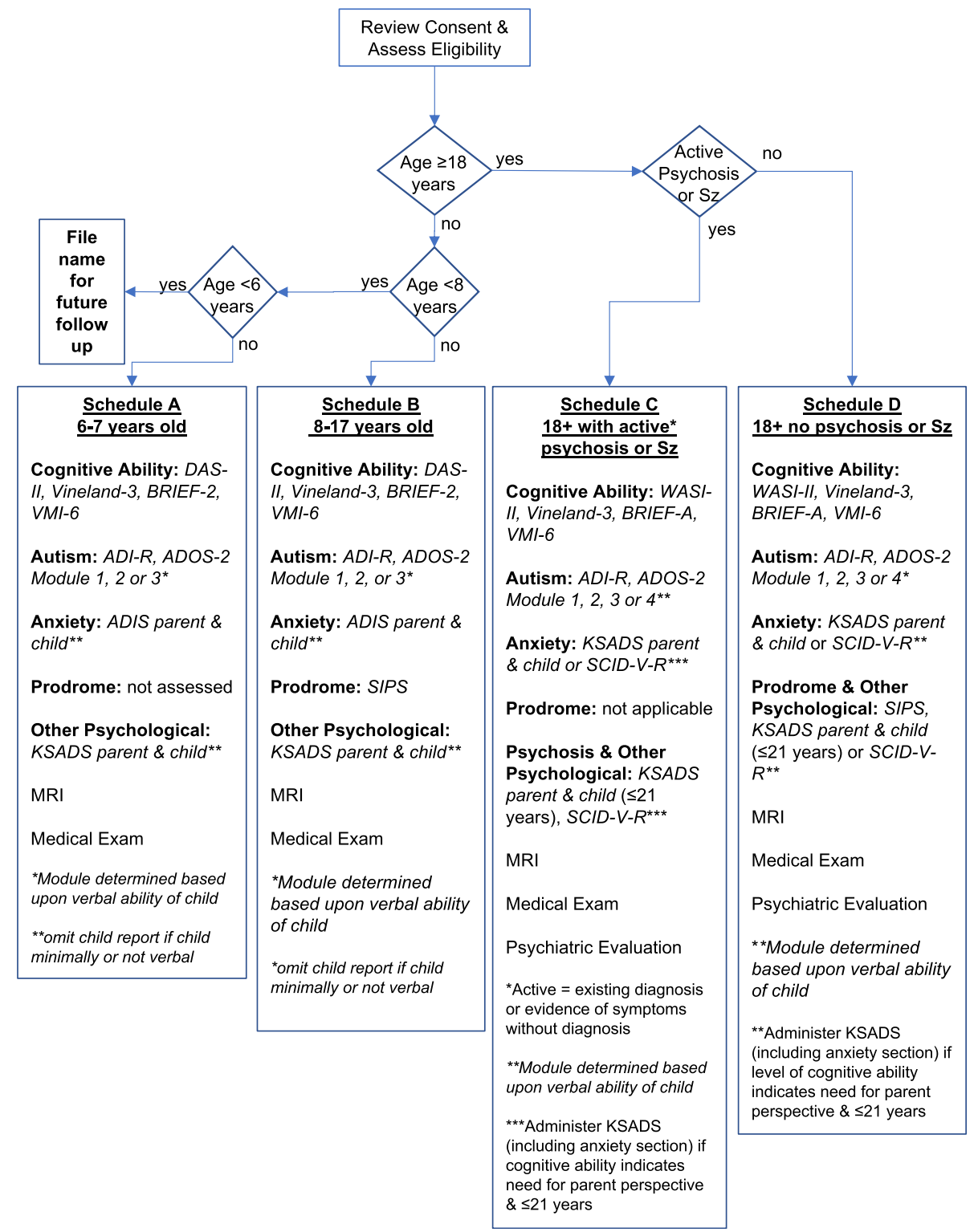

Fig. 1 Process flow diagram of the study protocol for phenotyping of 3q29 deletion syndrome. Assessment administration is determined based upon participant age, verbal ability, and evidence of active psychosis (defined as existing diagnosis of schizophrenia or evidence of psychosis without diagnosis). For participants under 18 years, the anxiety and autism sections of the KSADS are omitted to avoid overlap with the ADIS and ADI-R/ADOS-2. In this age group, child versions of the KSADS and ADIS are administered when the child's communication ability and emotional status allow engagement in the interview. Participants 18-21 years are administered the KSADS (including anxiety section) in lieu of the SCID-V-R, if their level of communication ability and emotional status suggest the need for parental perspective. Sz= Schizophrenia

any recommendations for ongoing care. Within a month of the study visit, the family also receives a comprehensive written report summarizing the results from each evaluation, and study contact information is provided in case there are concerns or questions. Following the visit, families are sent a feedback questionnaire via REDCap to enable further improvement of our process.

Family lodging and travel expenses are arranged and paid for by the study, and each participating family member receives an honorarium for the time involved in participation.

\section{Data management and analysis}

Family information, study questionnaires, medical exam data, photos, and recordings are stored in REDCap. The standardized study instruments are modeled in a local software system housed at the Marcus Autism Center, called DEX (Marcus Data Exchange). Modeling refers to 
the creation of the data structure for a given assessment/form and the accompanying platform to facilitate data entry (i.e., virtual form). The instruments are modeled following the National Database for Autism Research (NDAR)'s data dictionary guidelines, for future export from DEX into NDAR. All data entered into REDCap are double checked for accuracy. DEX follows a dual data entry system with automatic data checks, to ensure accurate data entry.

\section{Discussion}

The present paper describes the study protocol for a prospective study of the behavioral and clinical phenotype associated with 3q29 deletion syndrome. Systematic assessment of neuropsychiatric phenotypes has several advantages. First, consistent evaluation of the neurodevelopmental effects of the deletion can be used to establish standards of clinical care, including recommendations for early screening and intervention services, which may affect both short and long-term outcomes and management of the disorder, and are of high importance to families. It also establishes a baseline against which to measure the effects of any future therapeutic interventions.

Second, standardized assessment of all participants along the same four dimensions permits evaluation of whether there is an association among behavioral phenotypes or whether the phenotype arises as the result of a "core lesion" that primes the individual to develop a specific outcome. For example, an associated phenotypes hypothesis predicts that the presence and severity of each dimension of the phenotype is associated with the others (e.g., lower IQ is associated with higher anxiety and more compromised social functioning, which in turn are associated with more prodromal symptoms). Alternatively, a core lesion hypothesis suggests that an individual is primed to develop schizophrenia, autism, or anxiety, but that variable external forces at the behavioral or molecular level contribute to the specific phenotype. Evaluating these competing hypotheses provides important insights into potential mechanisms affecting the emergence of behavioral phenotypes in 3q29 deletion and other syndromes.

A third advantage of deep phenotyping work is that it can be leveraged for future studies aimed at linking behavioral and molecular data (neuronal phenotypes). Indeed, cellular and molecular data gathered in this study are being used to examine hypotheses including the effect of diploid status on the 3q29 neuronal phenotype, correlations between behavioral severity and neuronal morphology or function, and the presence of molecular phenotypes in inhibitory neurons. We will also have the opportunity to assess polygenic influences [35] and genetically dissect the relative contributions of interval genes to the multiple phenotypes expressed by carriers [36].
Finally, standardized assessment and systematic data capture of manifestations in 3q29 deletion syndrome, operationalized according to the protocol described here, allows for cross-comparison with other rare genetic syndromes, such as 16p11.2 duplication [5] and 22q11.2 deletion [37], where nearly identical instruments are deployed. As new syndromes with high risk for neuropsychiatric phenotypes are identified, it will become even more important to have harmonized systems for phenotypic evaluation. The protocol described here is one way forward.

Beyond the contributions of this study to describing the behavioral phenotype of 3q29 deletion and understanding its molecular and genetic basis, this study serves as an opportunity for family engagement, support, and education. For example, families report appreciating the opportunity to connect with professionals who are familiar with 3q29 deletion, its causes, and manifestations. Moreover, evaluations of the scope used in this study can be cost prohibitive for families seeking to have such testing independently. Although study evaluations are for research purposes, the detailed clinical report of the evaluations and follow up recommendations provided by our team of clinicians can support the development of interventions, inform IEPs, or help individuals qualify for services.

Continued evaluation of the neurodevelopmental effects of the deletion require that future studies follow individuals over time to document aspects of the phenotype as they change (e.g., low BMI and failure-to-thrive) or emerge over time (e.g., schizophrenia). Such work is strengthened by our efforts to build partnerships between families and researchers, as well as establish an international network of research collaborators. With the paradigm presented here, we hope to advance our understanding of 3q29 deletion syndrome, which may in turn serve as an entry point into a general molecular mechanism of neuropsychiatric phenotypes such as schizophrenia and autism.

\section{Abbreviations}

ADHD: Attention deficit hyperactivity disorders; ADI-R: Autism diagnostic interview schedule, revised edition; ADIS-IV: Anxiety disorders interview schedule for DMS-IN; ADOS-2: Autism diagnostic observation schedule, 2nd edition; ASD: Autism spectrum disorder; BRIEF-2 (BRIEF-A): Behavioral rating inventory of executive functions, 2nd edition (adult version); CBCL/ABCL: Child (Adult) behavior checklists; CHOA: Children's Hospital of Atlanta; CNV: Copy number variant; CSIC: Center for Systems Imaging Core; DAS-II: Differential Ability Scales, 2nd edition; DSM-IV (DSM-V): Diagnostic and statistical manual of mental disorders, 4th edition (5th edition); K-SADS: Kiddie-schedule for affective disorders and schizophrenia; NAPLS: North American Prodrome Longitudinal Study; NRGR: NIMH Repository and genomics resource; SCID-5: Structured clinical interview for DSM-5 disorders; SCQ: Social communication questionnaire; SIPS: Structured interview for psychosis-risk syndromes; Sz: Schizophrenia; VMI: Beery-Buktenica developmental test of visual-motor integration, 6th edition; WASI: Wechsler abbreviated scale of intelligence, 2nd edition 


\section{Acknowledgements}

The authors wish to acknowledge the grace and generosity of our study participants.

Ethics approval and consent to participate: This study has been approved by Emory University's Institutional Review Board (IBB00064133; IRB00088012). All subjects have informed consent prior to participating in this protocol.

\section{Funding}

This study is funded by NIH (1 R01 MH110701). Funding for the study was based on independent peer review. The funding body had no role in the design of the study, nor in the collection, analysis and interpretation of data. The funding body did not participate in writing of this manuscript.

\section{Availability of data and materials}

Data sharing is not applicable to this article as no datasets were generated or analyzed during the current study. Data generated in the course of this study will be publicly available. De-identified data on phenotypes will be deposited in the NIMH Data Archive (NDAR, https://ndar.nih.gov) and be publicly available to qualified investigators. De-identified biological samples will be deposited in the NIMH Repository and Genomics Resource (NGRG, https://www.nimhgenetics.org) and after a short embargo, be available to qualified investigators. Additional de-identified data not available through either of these sources will be made available from the authors upon reasonable request and with institutional IRB approval.

\section{Authors' contributions}

MMM and JGM coordinated the overall design of the study protocol and were major contributors in writing the manuscript. LB contributed expertise in evaluation of anxiety in pediatric patients with intellectual disability and autism, and evaluates patients using standardized instruments. JFC and EW contributed expertise in evaluation of active psychosis and prodromal features in pediatric and adult patients. RAE, KCBG, and DMN contribute expertise in evaluation of prodromal features and other psychiatric disorders, and conduct evaluations using the SIPS, KSADS, and SCID. MG and RLSR contribute expertise in medical genetics and conduct medical history interviews and physical exams. CK and CAS contribute expertise in evaluation of the autism phenotype, and conduct evaluations using the ADOS and ADI-R. CAS also contributes expertise in evaluating intellectual disability and adaptive behavior and evaluates patients. AP contributes expertise in database management, database capture of information, and expertise in design of REDCap instruments. SS and LL contribute expertise in collection and analysis of MRI data. All authors read and approved the final manuscript.

\section{Competing interests}

$\mathrm{COI}$ - CS is co-author on Vineland-3. The remaining authors declare they have no competing interests.

\section{Publisher's Note}

Springer Nature remains neutral with regard to jurisdictional claims in published maps and institutional affiliations.

\footnotetext{
Author details

${ }^{1}$ Department of Human Genetics, Emory University School of Medicine, Whitehead Research Building, 615 Michael Street, Suite 300, Atlanta, GA 30322, USA. 'Department of Pediatrics, Emory University School of Medicine, 1920 Briarcliff Road NE, Atlanta, GA 30322, USA. ${ }^{3}$ Marcus Autism Center, 1920 Briarcliff Road NE, Atlanta, GA 30322, USA. ${ }^{4}$ Emory Autism Center, 1551 Shoup Court, Atlanta, GA 30322, USA. ${ }^{5}$ Department of Psychiatry and Behavioral Sciences, Emory University School of Medicine, 12 Executive Park Drive, 2nd floor, Atlanta, GA 30329, USA. ${ }^{6}$ Department of Psychology, Emory University, 36 Eagle Row, Atlanta, GA 30322, USA. ${ }^{7}$ Department of Epidemiology, Rollins School of Public Health, Emory University, Atlanta, GA 30322, USA.

Received: 2 March 2018 Accepted: 22 May 2018 Published online: 08 June 2018

\section{References}

1. Ramoni RB, Mulvihill JJ, Adams DR, Allard P, Ashley EA, Bernstein JA, Gahl WA, Hamid R, Loscalzo J, McCray AT, et al. The undiagnosed diseases
}

network: accelerating discovery about health and disease. Am J Hum Genet. 2017;100(2):185-92.

2. Malhotra D, Sebat J. CNVs: harbingers of a rare variant revolution in psychiatric genetics. Cell. 2012;148(6):1223-41.

3. Landrum MJ, Lee JM, Riley GR, Jang W, Rubinstein WS, Church DM, Maglott DR. ClinVar: public archive of relationships among sequence variation and human phenotype. Nucleic Acids Res. 2014;42(Database issue):D980-5.

4. Simons Vip Consortium. Simons Variation in Individuals Project (Simons VIP): a genetics-first approach to studying autism spectrum and related neurodevelopmental disorders. Neuron. 2012;73(6):1063-7.

5. D'Angelo D, Lebon S, Chen Q, Martin-Brevet S, Snyder LG, Hippolyte L, Hanson E, Maillard AM, Faucett WA, Mace A, et al. Defining the effect of the 16p11.2 duplication on cognition, behavior, and medical comorbidities. JAMA psychiatry. 2016;73(1):20-30.

6. Green Snyder L, D'Angelo D, Chen Q, Bernier R, Goin-Kochel RP, Wallace AS, Gerdts J, Kanne S, Berry L, Blaskey L, et al. Autism Spectrum disorder, developmental and psychiatric features in 16p11.2 duplication. J Autism Dev Disord. 2016;46(8):2734-48.

7. Stefansson H, Meyer-Lindenberg A, Steinberg S, Magnusdottir B, Morgen $\mathrm{K}$, Arnarsdottir S, Bjornsdottir G, Walters GB, Jonsdottir GA, Doyle OM, et al. CNVs conferring risk of autism or schizophrenia affect cognition in controls. Nature. 2014;505(7483):361-6.

8. Sanders SJ, He X, Willsey AJ, Ercan-Sencicek AG, Samocha KE, Cicek AE, Murtha MT, Bal VH, Bishop SL, Dong S, et al. Insights into autism Spectrum disorder genomic architecture and biology from 71 risk loci. Neuron. 2015;87(6):1215-33.

9. Willatt L, Cox J, Barber J, Cabanas ED, Collins A, Donnai D, FitzPatrick DR Maher E, Martin H, Parnau J, et al. 3q29 microdeletion syndrome: clinical and molecular characterization of a new syndrome. Am J Hum Genet. 2005; 77(1):154-60.

10. Ballif BC, Theisen A, Coppinger J, Gowans GC, Hersh JH, Madan-Khetarpal S, Schmidt KR, Tervo R, Escobar LF, Friedrich CA, et al. Expanding the clinical phenotype of the 3q29 microdeletion syndrome and characterization of the reciprocal microduplication. Mol Cytogenet. 2008;1:8.

11. Cobb W, Anderson A, Turner C, Hoffman RD, Schonberg S, Levin SW. 1.3 Mb de novo deletion in chromosome band 3 q29 associated with normal intelligence in a child. European J Med Genet. 2010;53(6):415-8.

12. Glassford MR, Rosenfeld JA, Freedman AA, Zwick ME, Mulle JG. Novel features of 3q29 deletion syndrome: results from the $3 q 29$ registry. Am J Med Genet A. 2016;170a(4):999-1006.

13. Mulle JG. The $3 q 29$ deletion confers $>40$-fold increase in risk for schizophrenia. Mol Psychiatry. 2015;20(9):1028-9.

14. Mulle JG, Dodd AF, McGrath JA, Wolyniec PS, Mitchell AA, Shetty AC, Sobreira NL, Valle D, Rudd MK, Satten G, et al. Microdeletions of $3 q 29$ confer high risk for schizophrenia. Am J Hum Genet. 2010;87(2):229-36.

15. Silverman W, Albano A. The Anxiety Disord Interview Schedule for ChildrenIV (Child and Parent versions). San Antonio: Graywind Publications; 1996.

16. Rutter M, LeCouteur A, Lord C. Autism Diagnostic Interview, Revised. Los Angeles: Westerm Psychological Service; 2003.

17. Lord C, Risi S, Lambrecht L, Cook EH Jr, Leventhal BL, DiLavore PC, Pickles A, Rutter M. The autism diagnostic observation schedule-generic: a standard measure of social and communication deficits assocated with the spectrum of autism. J Autism Dev Disord. 2000;30(3):205-23.

18. Achenbach T, Rescorla L. Manual for the ASEBA Preschool Forms \& Profiles. In. Burlington: University of Vermont, Center for Children, Youth, \& Families; 2000.

19. Achenbach T, Rescorla L. Manual for the ASEBA School-age Forms \& Profiles. Burlington: University of Vermont, Research Center for Children, Youth, \& Families; 2001.

20. Achenbach T, Rescorla L. Manual for the ASEBA Adult Forms \& Profiles. Burlington: University of Vermont: Research Center for Children, Youth, \& Families; 2003.

21. Constantino J, Gruber C. Social responsiveness scale, 2nd edition [manual] Torrance: Western Psychological Services; 2012.

22. Rutter M, Bailey A, Lord C. The social communication questionnaire [manual]. Torrance: Western Psychological Services; 2003.

23. Ehlers S, Gillberg C, Wing L. A screening questionnaire for Asperger syndrome and other high-functioning autism spectrum disorders in school age children. J Autism Dev Disord. 1999;29(2):129-41.

24. Elliott CD. Differential abilities scales. 2nd ed. San Antonio: Harcourt Assessment; 2007.

25. Wechsler D. Wechsler abbreviated scale of intelligence. 2nd ed. Bloomington: Pearson; 2011. 
26. Sparrow S, Cicchetti D, Saulnier C. Vineland Adaptive Behavior Scales, 3rd ed. (Comprehensive Parent/Caregiver Interview). Circle Pines: American Guidance Service; 2016.

27. Beery K, Beery N. The Beery-Buktenica Visual Motor Integration Test. 6th ed. Pearson: Bloomington; 2010.

28. Gioia G, Isquith P, Guy S, Kenworthy L. Behavior Rating Inventory of Executive Function, 2nd ed. Professional Manual. Lutz: PAR; 2015.

29. Roth $R$, Isquith $P$, Gioia G. Behavior Rating Inventory of Executive FunctionsAdult Version (BRIEF-A) Professional Manual. Lutz: PAR, Inc; 2005.

30. Miller TJ, McGlashan TH, Rosen JL, Cadenhead K, Cannong T, Ventura J, McFarlane W, Perkins DO, Pearlson GD, Woods SW. Prodromal assessment with the structured interview for prodromal syndromes and the scale of prodromal symptoms: predictive validity, interrater reliability, and training to reliability. Schizophr Bull. 2003;29(4):703-15.

31. Addington J, Cadenhead KS, Cornblatt BA, Mathalon DH, McGlashan TH, Perkins DO, Seidman $L$, Tsuang MT, Walker EF, Woods SW, et al. North American Prodrome longitudinal study (NAPLS 2): overview and recruitment. Schizophr Res. 2012;142(1-3):77-82.

32. Kaufman J, Birmaher B, Brent D, Rao U, Ryan N. Schedule for affective disorders and schizophrenia for school aged children (6-18 years) - lifetime version (K-SADS-PL): initial reliability and validity data. J Am Acad Child Adoles Psychiatry. 1996;36(7):980-8.

33. First M, Williams J, Karg R, Spitzer R. User's guide for the structured clinical interview for DSM-5 disorders, research version (SCID-5-RV). Arlington: American Psychiatric Association; 2015.

34. Loewy RL, Pearson R, Vinogradov S, Bearden CE, Cannon TD. Psychosis risk screening with the prodromal questionnaire-brief version (PQ-B). Schizophr Res. 2011;129(1):42-6.

35. Tansey KE, Rees E, Linden DE, Ripke S, Chambert KD, Moran JL, McCarroll SA, Holmans P, Kirov G, Walters J, et al. Common alleles contribute to schizophrenia in CNV carriers. Mol Psychiatry. 2016;21 (8):1085-9.

36. Lopez-Rivera E, Liu YP, Verbitsky M, Anderson BR, Capone VP, Otto EA, Yan Z, Mitrotti A, Martino J, Steers NJ, et al. Genetic drivers of kidney defects in the DiGeorge syndrome. N Engl J Med. 2017;376(8):742-54.

37. Jonas RK, Jalbrzikowski M, Montojo CA, Patel A, Kushan L, Chow CC, Vesagas T, Bearden CE. Altered brain structure-function relationships underlie executive dysfunction in 22q11.2 Deletion Syndrome. Mol Neuropsychiatry. 2015;1(4):235-46.

\section{Ready to submit your research? Choose BMC and benefit from:}

- fast, convenient online submission

- thorough peer review by experienced researchers in your field

- rapid publication on acceptance

- support for research data, including large and complex data types

- gold Open Access which fosters wider collaboration and increased citations

- maximum visibility for your research: over $100 \mathrm{M}$ website views per year

At BMC, research is always in progress.

Learn more biomedcentral.com/submissions 